\title{
Sick Sinus Syndrome Observed in a Patient with Cholinesterase Deficiency
}

\author{
Shioto Yasuda ${ }^{1}$, Mitsuhiro Fukata ${ }^{1}$, Taku Yokoyama ${ }^{1}$, Takeshi Arita ${ }^{1}$, Keita Odashiro ${ }^{1,2}$, \\ Toru Maruyama ${ }^{1,2}$, Yoichiro Hiramoto ${ }^{2}$ and Koichi Akashi ${ }^{1}$
}

\begin{abstract}
:
A 58-year-old woman complained of general fatigue and was diagnosed with sick sinus syndrome (SSS) by ambulatory electrocardiogram, which demonstrated sinus arrest at midnight and paroxysmal atrial fibrillation (AF) at nighttime. Since her plasma cholinesterase (ChE) activity had been persistently zero, she was diagnosed with ChE deficiency. She refused permanent pacemaker implantation, and treatment with positive chronotropic drugs is ongoing. A novel association of ChE deficiency with SSS is theoretically possible rather than coincident, considering that $\mathrm{ChE}$ plays a key role in cholinergic influences on the sinus node leading to sinus bradyarrhythmia and on the atria, causing vagally mediated AF.
\end{abstract}

Key words: cholinesterase deficiency, sick sinus syndrome, chronotropism

(Intern Med 58: 809-812, 2019)

(DOI: 10.2169/internalmedicine.1229-18)

\section{Introduction}

Cholinesterase (ChE)-metabolizing acetylcholine (ACh) is an important and ubiquitous enzyme for cholinergic neurotransmission and some other physiological functions. ChE consists of two enzymes: acetylcholinesterase (AChE) and butyrylcholinesterase (BChE). AChE plays a crucial role in the exertion of cholinergic effects on the cardiac conduction system, but the role of $\mathrm{BChE}$ in cardiac phenotype is unclear.

We experienced a case of ChE deficiency demonstrating sick sinus syndrome (SSS) and short paroxysms of atrial fibrillation (AF) associated with a relatively slow ventricular response, and the rare combination of SSS and ChE deficiency is discussed.

\section{Case Report}

A 58-year-old woman with a history of ChE deficiency complained of general fatigue several years earlier. She had a family history in her father demonstrating an episode of prolonged action of succinylcholine administered in appendectomy. She was informed of a ChE deficiency when she underwent a blood examination at a health screening when she was 34 years old. However, she had no specific health problems thereafter. She experienced syncope during bathing when she was 40 years old and was diagnosed with SSS by ambulatory electrocardiogram (ECG). On her first visit to our hospital in 2011, the results of her plasma chemistry were unremarkable except for a nominal loss of plasma ChE activity. The serum potassium concentration remained normal, and her thyroid function was within the reference range. Her plasma ChE activity has been persistently and nominally zero thereafter.

Standard ECG showed sinus bradycardia without additional abnormalities as demonstrated in Fig. 1. Transthoracic ultrasound cardiogram (UCG) demonstrated a normal left ventricular function without any structural heart diseases. Ambulatory ECG performed without any medication demonstrated 69,980 total heart beats per day, and the daily mean heart rate (HR) was 49 beats per minute (bpm) as shown in the Table.

We made a diagnosis of SSS and recommended permanent pacemaker implantation. However, she refused it emphatically due to anesthetic problems and instead desired drug therapy. We obtained her informed consent with respect to medication as second-line therapy for SSS. Atropine sul-

${ }^{1}$ Heart Center, Kyushu University Hospital, Japan and ${ }^{2}$ Department of Medicine, Koga Central Hospital, Japan Received: March 22, 2018; Accepted: July 4, 2018; Advance Publication by J-STAGE: November 19, 2018 Correspondence to Dr. Toru Maruyama, maruyama@artsci.kyushu-u.ac.jp 


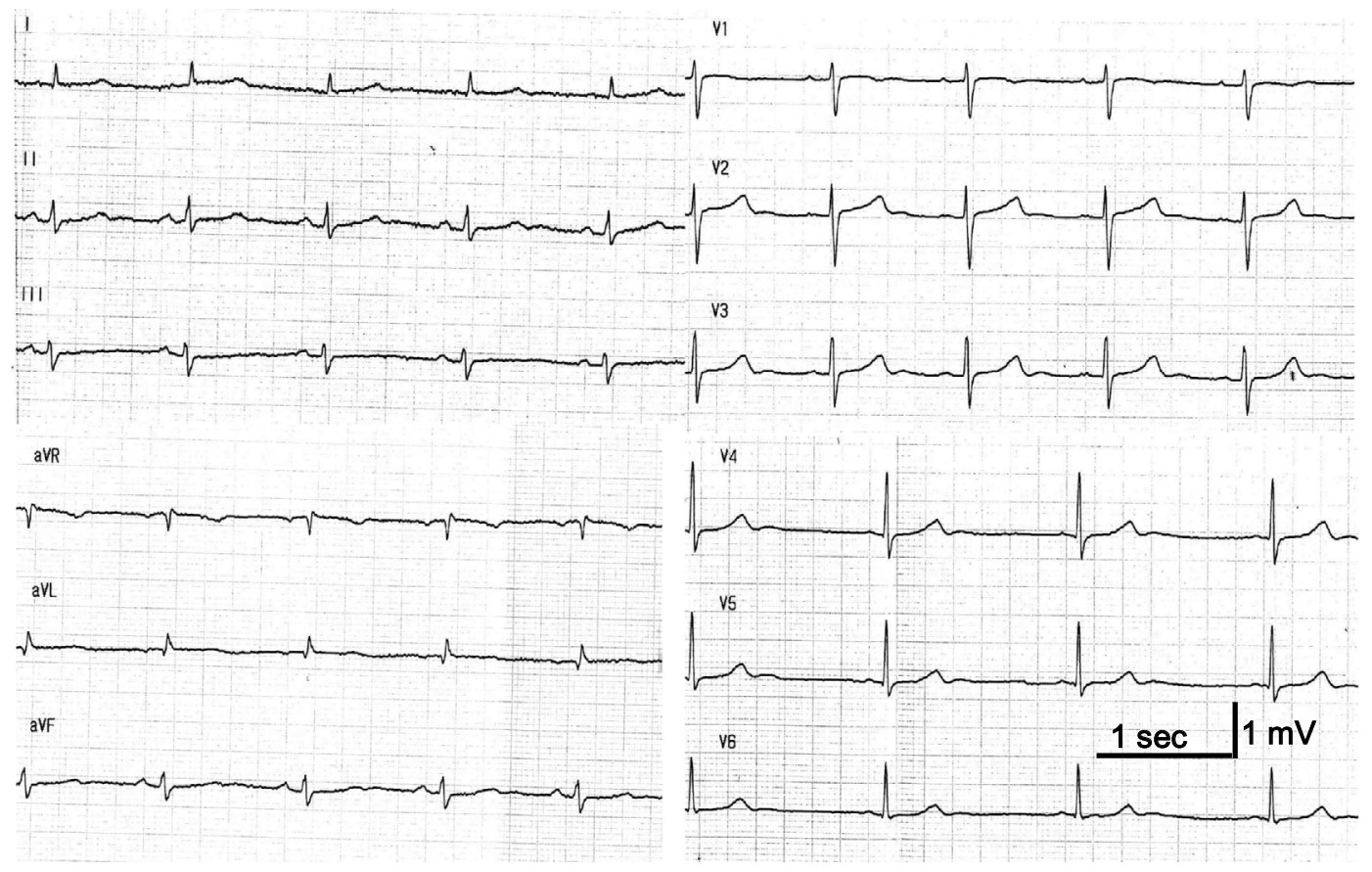

Figure 1. ECG showed sinus rhythm, and the mean heart rate was 54 beats per minutes. The heart rate gradually decreased over a short period of time.

Table. Results of Daily Heart Rate Trend Evaluated by Ambulatory Monitoring.

\begin{tabular}{lcccc}
\hline \multicolumn{1}{c}{ Drug } & $\begin{array}{c}\text { Hourly mean HR (bpm) } \\
\text { at daytime (7 AM to 8 PM) }\end{array}$ & $\begin{array}{c}\text { Hourly mean HR (bpm) } \\
\text { at nighttime (8 PM to 7 AM) }\end{array}$ & $\begin{array}{c}\text { Total heart beats } \\
\text { per day }\end{array}$ & $\begin{array}{c}\text { Mean heart rate } \\
\text { (bpm) }\end{array}$ \\
\hline No medication & $50-66$ & $30-43$ & 69,980 & 49 \\
Atropine sulfate (1.0 mg per day) & $51-84$ & $32-70$ & 80,640 & 56 \\
Transdermal tulobuterol (2.0 mg per sheet) & - & - & - & - \\
Amezinium metilsulfate (10 mg per day) & $49-82$ & $30-69$ & 79,540 & 55 \\
\hline
\end{tabular}

Ambulatory monitoring was not performed when transdermal tulobuterol was administered.

fate (1.0 mg per day) was administered but terminated due to photophobia. Ambulatory monitoring under the prescription of atropine sulfate converted junctional rhythm to sinus rhythm, and the maximum total number of heart beats was 80,640 beats per day (Table). A prescription of a transdermal patch containing tulobuterol $(2.0 \mathrm{mg}$ per sheet), a longacting bronchodilator, was attempted but renounced due to skin discomfort. We then prescribed amezinium metilsulfate (20 mg per day), an inhibitor of both the reuptake and inactivation of noradrenaline, and reduced the dosage $(10 \mathrm{mg}$ per day) due to palpitation.

Current ambulatory monitoring during normal daily activity showed 79,540 total heart beats per day, and the daily mean HR was $55 \mathrm{bpm}$. An HR trend graph is shown in Fig. 2A. The hourly mean HR evaluated by ambulatory monitoring ranged from 30 to $69 \mathrm{bpm}$ at night (8 PM to 7 $\mathrm{AM}$ ) and from 49 to $82 \mathrm{bpm}$ in the day (7 $\mathrm{AM}$ to $8 \mathrm{PM}$ ), respectively (Table). As specific events, nocturnal sinus arrest showing the longest pause of 4.4 seconds was observed at 2:40 AM, as indicated in Fig. 2B. Paroxysms of atrial fibrillation $(\mathrm{AF})$ were detected twice by ambulatory monitoring (8:40 PM and 5:10 AM). Real-time recording of the onset of AF detected in the evening is shown in Fig. 2C. The duration of these paroxysms was typically short (84 seconds and 14 seconds, respectively). The respective maximum, minimum and mean ventricular rates during AF were 83, 48 and $51 \mathrm{bpm}$, indicating that the ventricular rate during $\mathrm{AF}$ was not rapid and thereby implying vagal predominance. However, a long pause immediately after the restoration of sinus rhythm was not observed. Sinoatrial but not atrioventricular (AV) block was observed during sinus rhythm at night. HR variability was not analyzed due to nocturnal sinus bradyarrhythmia and frequent supraventricular premature beats during the day.

She has experienced no syncope or palpitation under the titration of amezinium metilsulfate.

\section{Discussion}

Although $\mathrm{AChE}$ and $\mathrm{BChE}$ are reported to influence the incidence and mortality of coronary artery disease $(1,2)$, the association of ChE deficiency with SSS is not clear. AChE plays a key role in cholinergic neurotransmission, and $\mathrm{BChE}$ plays a supplementary role to AChE. ChE deficiency ob- 


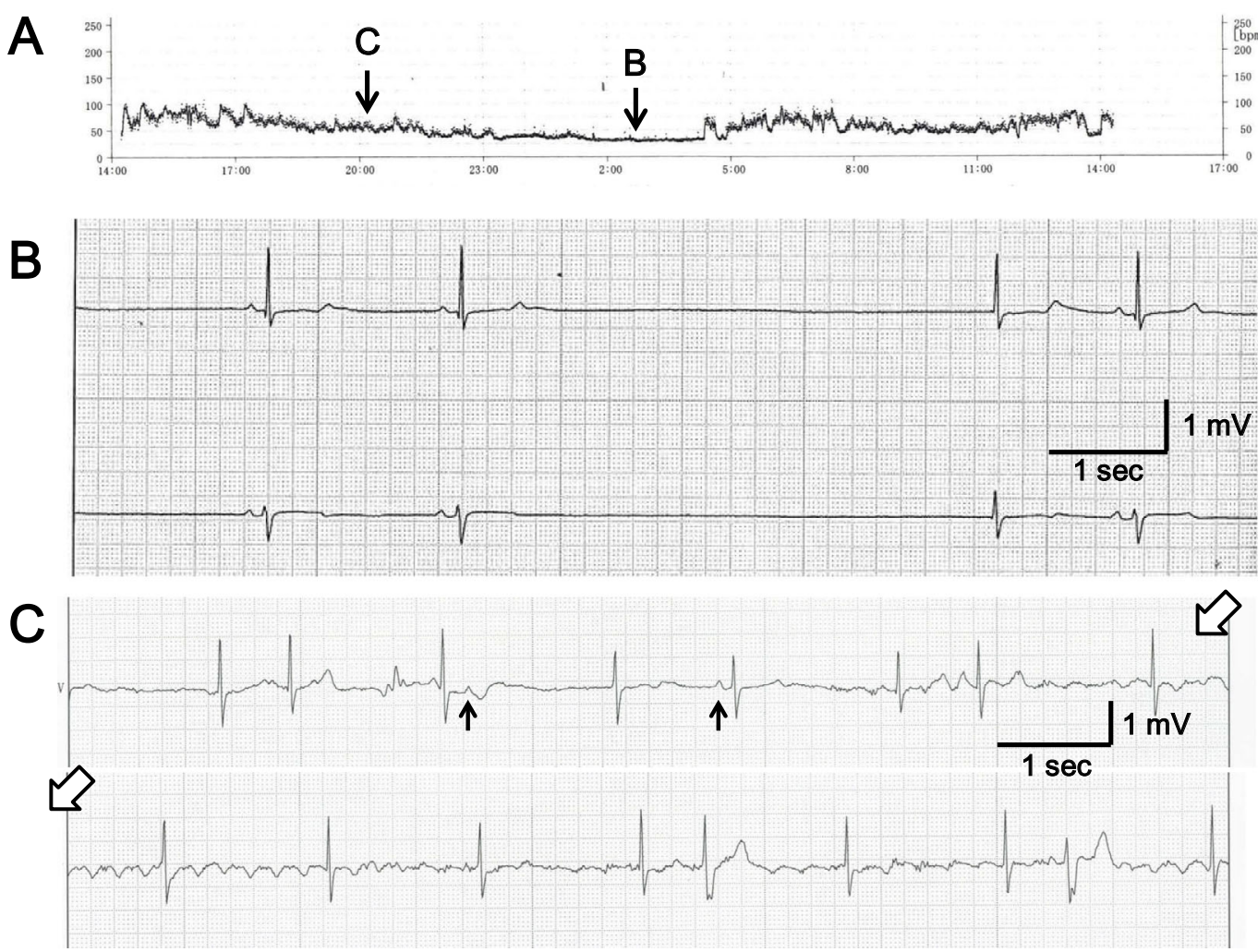

Figure 2. Ambulatory ECG monitoring under treatment with amezinium metilsulfate. A: Amezinium metilsulfate (10 $\mathrm{mg}$ per day) was given, and the heart rate showed bradycardia and sinus arrest during the nocturnal period. B: Real-time recording at the time (B) in A showed sinus arrest at midnight. C: Real-time recording at the time (C) in A showed paroxysmal atrial fibrillation (AF) in the evening. The atrial activity observed in the upper column is indicated by arrows. The upper and lower columns indicate consecutive recordings.

served in apparently healthy individuals manifests as the loss of BChE activity. Considering the nominal loss of ChE activity and normal liver function (AST of 20 and ALT of 17) observed in the present patient, our case is suspected to be a silent variant (3).

Whether SSS is a novel expression of ChE deficiency or these two clinical entities are coincident is controversial. ChE deficiency may enhance or prolong cholinergic transmission by inhibiting ACh hydrolysis and upregulating the local ACh level, leading to negative cardiac chronotropism (4). Although she was unable to tolerate them for long, cAMP-mediated positive chronotropic agents (tulobuterol and amezinium) and vagolytic atropine were effective to some degree in increasing the sinus rate. Such pharmacologic responses suggest that her sinus node is functionally suppressed. Nocturnal sinus bradyarrhythmia was evident, but AV block was not recorded by ambulatory monitoring (Fig. 2). This is considered to reflect heterogeneous autonomic innervation of the human cardiac conduction system. The density of the innervation is reported to be highest in the sinus node, and the ChE activity is greatest in the sinus node. This ChE activity has been reported to decrease in the AV node (5). This immunohistochemical study supports the notion that the sinus node is more susceptible to ChE deficiency than the AV node.
AF observed in this case showed several characteristics. Short paroxysms of nonvalvular AF occurring at night (8 $\mathrm{PM}$ to $7 \mathrm{AM}$ ) with low ventricular responses $(83 \mathrm{bpm}$ at maximum) might be related to dominant vagal activity (6). Given that observed AF is suspected to be mediated vagally, enhanced cholinergic transmission may influence the sinus node and atria in this case. Indeed, potent cholinesterase inhibition induced by intravenous edrophonium is reported to decrease the HR in anesthetized patients dosedependently (7).

However, we should be careful of concluding that the present patient's ChE deficiency is linked directly to her arrhythmogenesis. Most patients with silent ChE activity shown no serious health problems, with normal ECG and UCG findings (8), suggesting that some mechanisms compensate for the ChE deficiency. Animal models are a useful reference in the present case; for example, the fluorescent intensity of near-infrared dye-labeled BChE is actually detected in the heart of BChE-knockout mice, a model of human ChE deficiency (9). Another possible mechanism underlying the backup of $\mathrm{BChE}$ deficiency is the counterregulation of $\mathrm{ChE}$ activity; indeed, $\mathrm{BChE}$ knockdown has been shown to cause the upregulation of the activities of AChE and other kinases (e.g., protein kinase $\mathrm{C}$ and extracellular signal-regulated kinase) mediated by transcriptional 
factors (10). These findings indicate crosstalk between $\mathrm{AChE}$ and $\mathrm{BChE}$ in at least pluripotent cell lines.

Although we have no concrete explanation for the combination of ChE deficiency and the premature onset of SSS in the present case, such compensatory mechanisms underlying backup silent ChE may have malfunctioned or declined agedependently in this case.

The authors state that they have no Conflict of Interest (COI).

\section{Acknowledgement}

We thank Mr. Shinji Tagaya for the concise retrieval of the ambulatory monitoring data.

\section{References}

1. Scacchi R, Ruggeri M, Corbo RM. Variation of the butyrylcholinesterase (BChE) and acetylcholinesterase (AChE) genes in coronary artery disease. Clin Chim Acta 412: 1341-1344, 2011.

2. Sun L, Qi X, Tan Q, Yang H, Qi X. Low serum butyrylcholinesterase activity as a prognostic marker of mortality associates with poor cardiac function in acute myocardial infarction. Clin Lab 62: 1093-1099, 2016.

3. Yen T, Nightingale BN, Burns JC, Sullivan DR, Stewart PM. Butyrylcholinesterase (BChE) genotyping for post-succinylcholine apnea in an Australian population. Clin Chem 49: 1297-1308, 2003.
4. Cuartero M, Ortuño JA, García MS, García-Cánovas F. Assay of acetylcholinesterase activity by potentiometric monitoring of acetylcholine. Anal Biochem 421: 208-212, 2012.

5. Chow LT, Chow SS, Anderson RH, Gosling JA. Autonomic innervation of the human cardiac conduction system: changes from infancy to senility - an immunohistochemical and histochemical analysis. Anat Res 264: 169-182, 2001.

6. Rawles JM. What is meant by a 'controlled' ventricular rate in atrial fibrillation? Br Heart J 63: 157-161, 1990.

7. Deschamps A, Backman SB, Novak V, Plourde G, Fiset P, Chartrand D. Effects of the anticholinesterase edrophonium on spectral analysis of heart rate and blood pressure variability in humans. J Pharmacol Exp Ther 300: 112-117, 2002.

8. Manoharan I, Boopathy R, Darvesh S, Lockridge O. A medical health report on individuals with silent butyrylcholinesterase in the Vysya community of India. Clin Chim Acta 378: 128-135, 2007.

9. Duysen EG, Lockridge O. Whole body and tissue imaging of the butyrylcholinesterase knockout mouse injected with near infrared dye labeled butyrylcholinesterase. Chem Bio Interact 175: 119124, 2008.

10. Bodur E, Layer PG. Counter-regulation of cholinesterases: differential activation of PKC and ERK signaling in retinal cells through BChE knockdown. Biochimie 93: 469-476, 2011.

The Internal Medicine is an Open Access journal distributed under the Creative Commons Attribution-NonCommercial-NoDerivatives 4.0 International License. To view the details of this license, please visit (https://creativecommons.org/licenses/ by-nc-nd/4.0/).

(C) 2019 The Japanese Society of Internal Medicine Intern Med 58: 809-812, 2019 